\title{
Prevalence of abnormal kidney function and 90- days outcomes among patients admitted on medical wards of Masaka Regional Referral Hospital in Uganda: a prospective study
}

SSenabulya F. Ronny ( $\square$ frssenna@gmail.com )

Makerere University College of Health Sciences

Nankabirwa I. Joaniter

Makerere University College of Health Sciences

Kalyesubula Robert

Makerere University College of Health Sciences

Wandera Bonnie

Makerere Lung Institute

Kirenga Bruce

Makerere University College of Health Sciences

Kayima James

Makerere University College of Health Sciences

Ocama Posiano

Makerere University College of Health Sciences

\section{Bagasha Peace}

Makerere University College of Health Sciences

\section{Research Article}

Keywords: Abnormal kidney function, Regional Referral Hospital, 90 days outcome

Posted Date: February 16th, 2022

DOI: https://doi.org/10.21203/rs.3.rs-1322172/v1

License: (9) This work is licensed under a Creative Commons Attribution 4.0 International License.

Read Full License

Version of Record: A version of this preprint was published at BMC Nephrology on July 7th, 2022. See the published version at https://doi.org/10.1186/s12882-022-02865-w. 


\section{Abstract}

Background: Kidney disease burden is raising worldwide, raising a need to closely monitor high risk populations for swift response. However, there is limited data on the prevalence of abnormal kidney function and outcomes among patients admitted at regional referral health facilities in Uganda which are the first points of care for most patients in the country. We determined the prevalence of abnormal kidney function and 90-days outcomes among patients admitted on medical ward of Masaka Regional Referral Hospital.

Methods: This was a prospective study conducted among adult patients admitted on medical wards between September 2020 to March 2021. Patients were enrolled if they met the following inclusion criteria: a) $\geq 18$ years of age, 2) provided written informed consent, 3) had phone contacts to help with patient follow up. Spot urine sample was collected to assess for proteinuria and a blood sample was collected to assess serum creatinine levels. Abnormal kidney function was defined as decreased estimated glomerular filtration rate $<90 \mathrm{ml} /$ minute $/ 1.73 \mathrm{~m}^{2}$ or proteinuria of $\geq 1+$ on spot urine dipstick. Estimated glomerular filtration rate (eGFR) was calculated using the CKD-Epi formula. Patients with abnormal kidney function were followed up at 90 days post enrollment to determine their outcomes.

Results: Were enrolled 357 patients. The overall prevalence of abnormal kidney function was $32.8 \%$ (117/357), 08 (2.2\%) patients having proteinuria alone, 68 (19.1\%) having decreased eGFR alone, and 41 (11.5\%) having both proteinuria and decreased eGFR. The median age of 117 patients with abnormal kidney function was 52 (IQR 39-70) years and majority were males (54.7\%). Of the 117 patients with abnormal kidney function, 108 were followed up to 90-days. The 90 days mortality was $69.4 \%(75 / 108)$, and $13.0 \%(14 / 108)$ had progressed to chronic kidney disease.

Conclusions: The prevalence of abnormal kidney function was high and the many had adverse outcomes including death and progression to chronic kidney disease. To reduce on these adverse outcomes, patients at risk or with abnormal kidney function may benefit from basic screening and use of management protocols for kidney disease.

\section{Background}

The burden of kidney disease is rising worldwide, with estimates in 2017 at $29.5 \%$ compared to the $19.7 \%$ in 2007 (1). Mild abnormalities in kidney structure and function are associated with increased risk for complications \& mortality (2). Abnormal kidney function may present as acute kidney injury (AKI), acute kidney disease and disorders (AKD) or as chronic kidney disease (CKD). Kidney failure is end-stage of AKI ,AKD or CKD requiring treatment by dialysis or transplantation $(3,4)$. Therefore, early screening and identification of patients at risk or with early abnormal kidney function allows prompt management of patients before development of kidney failure related complications and mortality.

In Uganda, the general burden of kidney disease is not well documented, however, a few studies evaluating kidney function have been conducted in different settings including outpatient clinic settings 
(5), community-based setting (6) and among special populations at high risk of abnormal kidney function $(7,8)$. These studies have shown a generally high burden of abnormal kidney function in these settings. However, no studies have estimated the magnitude of abnormal kidney function and outcomes among patients admitted to regional referral hospitals in Uganda. Appreciating the burden in regional referral hospitals is important as this is the first point and sometimes the only point of care for most patients in the country. This study estimated the prevalence of abnormal kidney function and 90-days outcomes among patients admitted on medical wards of Masaka Regional Referral Hospital (RRH).

\section{Methods}

\section{Study design and setting:}

This was a prospective study conducted among adult patients admitted on the medical wards of Masaka $\mathrm{RRH}$. This is the largest government hospital in Masaka district, which is located in south central Uganda. The hospital provides both preventive and curative services for over two million people. This hospital has 330 bed capacity with the medical wards using up over $21 \%$ of the hospital beds. Medical wards admit patients with various medical conditions including but not limited to anemia, liver cirrhosis, heart failure, malaria, diabetes mellitus and hypertensive disorders. The hospital has no specialized nephrology clinic; however, it runs a daily general medical outpatient clinic, hypertension and diabetes mellitus clinics at least once a week.

\section{Study population and procedures:}

All adult patients admitted to the medical wards of Masaka RRH between September 2020 and November 2020 were consecutively screened for eligibility to join the study. Patients were enrolled if: 1) were aged 18 years and above; 2 ) were admitted on medical wards for at least 24 hours; 3 ) provided written informed consent. Patients were excluded if they had no phone contacts to allow follow-up. A detailed questionnaire was administered to all enrolled patients by study personnel. The questionnaire captured information on demographics and risk factors for disease. A venous blood sample and a midstream spot urine sample were collected from all patients using aseptic techniques. Approximately $4 \mathrm{mls}$ of venous blood was drawn from each patient into a syringe then placed into a red top vacutainer to assess for serum creatinine. All patients were provided with a sterile urine container and educated briefly about provision of $20 \mathrm{mls}$ of early morning mid-stream urine to assess for proteinuria. A follow up phone call was conducted for all patients meeting the definition of abnormal kidney function 90-days following the baseline assessment. Abnormal kidney function was defined as proteinuria of $\geq 1+$ or decreased eGFR of $<90 \mathrm{mls} /$ minute $/ 1.73 \mathrm{~m}^{2}$. Patients that were still alive were requested to return to the medical outpatient clinic for a repeat venous blood sample collection to measure the 90-day serum creatinine and spot urine dipstick testing for proteinuria. Outcomes of interest at the 90 day follow up included; 1) all-cause mortality, 2) CKD and, 3) no confirmed chronic kidney disease. Chronic kidney disease was defined as 
decreased eGFR $<60 \mathrm{mls} / \mathrm{min} / 1.73 \mathrm{~m}^{2}$ or proteinuria of $\geq+1$ both at baseline survey and after 90 -days at follow up.

\section{Laboratory evaluations}

The venous blood collected was centrifuged within one hour of collection to obtain serum. Sample analysis for serum creatinine was conducted in Masaka RRH laboratory using the Jaffe method traceable to an isotope dilution mass spectrometry (9). Urine samples were tested within 30 minutes of collection for proteinuria using a 10-parameter dipstick (Urinspect strips) manufactured by Artron laboratories.

\section{Data management and statistical analysis}

All data was entered into Microsoft office excel database, then exported to STATA version 13 software package for analysis. Continuous and discrete variables were summarized into means with standard deviations, medians and interquartile ranges (IQR). Categorical variables were summarized into frequencies and percentages. The eGFR was calculated using chronic kidney disease Epidemiology Collaboration (CKD-EPI) creatinine-based equation $(10,11)$. The prevalence of abnormal kidney function was calculated as the total number of patients with proteinuria of $\geq 1+$ or decreased eGFR of $<90 \mathrm{mls} / \mathrm{min} / 1.73 \mathrm{~m}^{2}$, divided by the total number of patients enrolled.

Proportion of patients who developed chronic kidney disease was calculated as number of patients with decreased eGFR of $<60 \mathrm{mls} / \mathrm{min} / 1.73 \mathrm{~m}^{2}$ or proteinuria of $\geq+1$ both at baseline survey and after 90 -days at follow up divided by total number of patients with abnormal kidney function. All-cause mortality at 90 days was calculated as the total number of patients reported as dead divided by the number of patients with abnormal kidney function who were followed up.

\section{Results}

\section{Characteristics of the study population}

Between September and November 2020, a total of 717 patients were screened for eligibility to join the study, of which 357 (49.8\%) were enrolled. Reason for exclusion of the 360 potential patients included lack of a phone contact to allow follow-up 327/360 (90.8\%), age < 18 years $32(8.9 \%)$, and $01(0.3 \%)$ refused to consent to participate in the study. The median age of the 356 patients enrolled was 47 (IQR 32-63) years, males constituted $52.9 \%$, and majority of had no or only primary education (75.9\%). Details of baseline characteristic are presented in table 1 below.

Table 1

Characteristics of the study participants 


\begin{tabular}{|c|c|}
\hline \multirow[t]{2}{*}{ Characteristic } & Proportions (\%) \\
\hline & $N=357$ \\
\hline \multicolumn{2}{|c|}{ Age categories: Median (IQR) } \\
\hline $18-35$ & $28(20-31)$ \\
\hline $36-59$ & $47(40-52)$ \\
\hline 60 years and above & $73(66-80)$ \\
\hline \multicolumn{2}{|l|}{ Sex } \\
\hline Female & $168(47.1)$ \\
\hline Male & $189(52.9)$ \\
\hline \multicolumn{2}{|l|}{ Tribe } \\
\hline Baganda & $241(67.5)$ \\
\hline Banyakitara & $52(14.6)$ \\
\hline Others ${ }^{1}$ & $64(17.98)$ \\
\hline \multicolumn{2}{|l|}{ Occupation } \\
\hline Civil/NGO servant & $20(5.6)$ \\
\hline Operate business & $73(20.5)$ \\
\hline Peasant farmer & $177(49.6)$ \\
\hline Unemployed & $49(13.7)$ \\
\hline Others ${ }^{2}$ & $38(10.6)$ \\
\hline \multicolumn{2}{|l|}{ Religion } \\
\hline Born again and SDA & $33(9.24)$ \\
\hline Catholic & $202(56.6)$ \\
\hline Moslem & $78(21.9)$ \\
\hline Protestant & $44(12.3)$ \\
\hline \multicolumn{2}{|l|}{ Level of Education } \\
\hline No education & $55(15.4)$ \\
\hline Primary & $216(60.5)$ \\
\hline Secondary & 65 (18.2) \\
\hline
\end{tabular}




\begin{tabular}{|lc|}
\hline Tertiary & $21(5.9)$ \\
\hline Marital status & $76(21.3)$ \\
\hline Divorced/separated & $148(41.4)$ \\
\hline Single & $58(16.3)$ \\
\hline Widow/widower & $75(21.0)$ \\
\hline Others': Acholi, Iteso, Basoga; Other $^{\mathbf{2}}$ : Carpenter, Builder and manual laborer
\end{tabular}

\section{Prevalence of abnormal kidney function}

Out of 357 patients enrolled, 117 (32.8\%) had abnormal kidney function. Details are shown in figure 1 below. Of the117 participants with abnormal kidney function, 68 (58.1\%) had decreased eGFR of < $90 \mathrm{mls} /$ minute $/ 1.73 \mathrm{~m}^{2}$ alone, $8(6.8 \%)$ had proteinuria $\geq 1+$ alone, and $41(35.1 \%)$ had both decreased eGFR and proteinuria $\geq 1+$. The median age of the 117 patients was 52 (IQR 39-70) years and 64 (54.7\%) were males. Known risk factors of kidney disease among the patients with abnormal kidney function included: 1) alcohol consumption 48 (41.0\%); 2) cigarette smoking 25 (21.4\%); 3) known hypertensive 39 (33.3\%); 4) known diabetics 22(19.3\%); and 5) HIV positive 32 (27.4\%).

\section{0-days outcomes of patients with abnormal kidney function}

Of the 117 patients with abnormal kidney function, 108 (92.3\%) were located at the 90-days follow up visit with only 9 (7.7\%) lost to follow-up. Of the 108 participants located, 75 were reported as deceased giving an all-cause mortality of $69.4 \%$. A total of 14 (13.0\%) patients had CKD and 19 (17.6\%) had no confirmed CKD suggesting possible resolution of disease. Of the 75 patients that died, $40(53.3 \%)$ had only decreased eGFR at baseline, 5 (6.7\%) had only proteinuria of $\geq 1+$ at baseline, and 30 (40\%) had both reduced eGFR and proteinuria of $\geq 1+$ at baseline.

Of the 14 patients with confirmed CKD, $06(42.9 \%)$ had only decrease eGFR of $<60 \mathrm{mls} /$ minute $/ 1.73 \mathrm{~m}^{2}$ at baseline, 01 (7.1\%) had only proteinuria $\geq 1+$ at baseline, and 07 (50\%) had both decreased eGFR of < $60 \mathrm{mls} /$ minute $/ 1.73 \mathrm{~m}^{2}$ and proteinuria $\geq 1+$ at baseline.

\section{Discussion}

As the overall burden of kidney disease increases, there is a need for increased screening, early identification and management of cases to prevent adverse outcomes from the disease processes. In this study, we estimated the prevalence of abnormal kidney function and 90-days outcomes among patients 
admitted on the medical wards in Masaka RRH between September and November 2020. We found that at least one in every three patients had abnormal kidney function with decreased eGFR contributing the highest proportion of the abnormal function. The 90-days mortality among the patients with abnormal kidney function was high with more than half of the patients dying within this period. In addition, progression to CKD was high among this population, with one in ten participants progressing to CKD. These results show that there is high burden of abnormal kidney function among patients admitted to medical wards at this regional referral facility in Uganda and highlight the need for mechanisms to screen for abnormal kidney function at these facilities to accelerate medical response.

We observed a high prevalence of abnormal kidney function in this study and given that the patients were admitted on the medical wards due to other disease conditions, the abnormal function observed could be due to multiple etiologies. Many of the patients with abnormal kidney function had known risk factors for kidney disease including advanced age, comorbidities including HIV, diabetes mellitus and hypertension, as well as risky lifestyles like smoking and alcohol consumption (12-14).

Irrespective of the original cause of abnormal kidney function, its high prevalence is of concern as this can easily progress to CKD, kidney failure and death in this population. Indeed, the observed prevalence is higher than what has been described previously in Uganda. A study by Nyende et al in 2020 estimated the prevalence of abnormal kidney function at $2.5 \%$ among HIV patients attending an out-patient clinic (15) and another study conducted in the general population which estimated the prevalence of abnormal eGFR at $19.8 \%$ (16). The differences observed in our estimates compared to these estimates could be mainly because of the differences in populations studied, different definitions of abnormal kidney function and the different methods used to determine eGFR.

Mortality among patients with abnormal kidney function was high at $69.4 \%$ thus more than half of the patients had died within 90-days. This was all cause mortality, hence could be accounted for by other illnesses including sepsis, advanced HIV/AIDS, uncontrolled diabetes mellitus and hypertensive complications. This high mortality is similar to findings from several studies; Machado et al, 2014 in Brazil, found a 30 -days mortality of $66 \%$ among patients with abnormal kidney function (Machado, Nakazone, \& Maia, 2014). A collaborative meta-analysis of kidney disease population cohorts showed a positive association between lower eGFR category and mortality (17) and during another pooled analysis of community-based studies including Atherosclerosis Risk in Communities Study (ARIC), the Cardiovascular Health Study (CHS), the Framingham Heart Study (FHS), and the Framingham Offspring Study (Offspring), subjects with CKD (eGFR: 15 to $60 \mathrm{ml} / \mathrm{min} / 1.73 \mathrm{~m} 2$ ) had a $36 \%$ excess risk for allcause mortality (18). Another prospective cohort study in Tanzania by found mortality rates of $56.6 \%$ after 3 months (19). These studies show that patients with abnormal kidney function are at high risk of death.

In our study the very high mortality was probably because most patients with CKD or kidney failure were not able to access prompt ideal care including hemodialysis or transplant for those who had kidney 
failure thus may have partly died of kidney failure related complications in addition to their primary comorbidities.

The incidence of CKD among the patients with abnormal kidney function was also high with one in every ten patients having CKD after 90-days of follow-up. CKD was confirmed using decreased eGFR of $<60$ $\mathrm{mls} /$ minute $/ 1.73 \mathrm{~m}^{2}$ and proteinuria of $\geq 1+$ both at baseline and after 90 -days during follow up. This is in agreement with findings of other studies about outcomes for patients with abnormal kidney function. A study among patients attending hypertension clinic of Mulago National Referral Hospital also found the prevalence of CKD was $17.2 \%$ after 90 -days of follow up (5). However finding of our study differ from findings of a prospective study conducted in the adult medical wards of a teaching hospital in Tanzania where a very high prevalence of CKD was found to be $69.5 \%$ after three months follow up of patients with reduced eGFR of $<60 \mathrm{ml} / \mathrm{min} / 1.73 \mathrm{~m}^{2}(19)$. The lower prevalence in our study could partly be related to the very high mortality for our study patients with decreased eGFR at baseline and traditional risk factors for CKD which a significant number of these patients had including diabetes mellitus, hypertension and HIV/AIDS.

The study is not without limitations. First, we only followed up only participants with abnormal kidney function thus we are unable to compare outcomes with those patients having normal kidney function. Although this may affect the mortality outcome in that mortality may be comparable among the two groups, we are confident the CKD findings would be higher in the two groups given that abnormal kidney function is in the pathway of CKD. Second, we never performed renal ultrasound scan which would enable us to diagnose more patients with CKD basing on renal structural abnormalities and lastly, our study was not powered to establish factors associated with 90-days outcomes at follow-up period due to the small sample size, thus one may urge that high mortality for patients with abnormal kidney function could have been attributed to other comorbid illnesses among some patients.

\section{Conclusion}

The prevalence of abnormal kidney function among patients admitted on medical wards of Masaka RRH was high and many of these patients had adverse outcomes including death and progression to CKD. Early screening of patients at risk or with early abnormal kidney function should be done to ensure swift management of patients before development of kidney failure related complications and mortality.

\section{Abbreviations}

CKD: Chronic kidney disease; CKD-EPI: Chronic Kidney Disease Epidemiology Collaboration; eGFR: Estimated glomerular filtration rate; IQR: Interquartile Range; RRH: Regional Referral Hospital; SCr: Serum Creatinine

\section{Declarations}




\section{Acknowledgements}

The authors thank everyone who participated in this study, the staff of Masaka RRH and Department of Internal Medicine Makerere University Kampala for all the support provided to conduct the study. This study was a dissertation for a master's degree of internal medicine.

\section{Authors' contributions}

SFR conceived the idea, collected data, interpreted the data \& drafted first version of manuscript. KR, NIJ, BP: Designed the study protocol, analyzed the data, interpreted the data, drafted first version of manuscript and approved the final version of the manuscript. KB, OP, KJ, BW: reviewed final draft of the work and manuscript.

\section{Funding}

The work was supported by MAKNCD research training program. Funding was provided by US NIHFogarty International Center grant titled "Training in non-communicable diseases, biomedical, clinical, epidemiological and Implementation science research to strengthen evidence-based interventions, policy, and control in Uganda (Grant \# D43TW011401)". The funders had no role in the study design, data collection and analysis, decision to publish or preparation of the manuscript.

\section{Availability of data and materials}

All data generated or analyzed during this study are included in the supplementary additional file 1.

\section{Ethics approval and consent to participate}

All methods in this study were performed in accordance with the World Medical Association declaration of Helsinki (Ethical Principles for Medical Research Involving Human Subjects). The study was approved by Makerere University School of Medicine, Research \& Ethics Committee approval No. REC REF 2020160 and got administrative approval from Masaka RRH. All patients who were enrolled in this study provided written informed consent. The consent process was administered in both English and local language determined by the preference of the patient.

\section{Consent for publication}

"Not applicable" 


\section{Competing interests}

The authors declare that they have no competing interests.

\section{References}

1. James SL, Abate D, Abate KH, Abay SM, Abbafati C, Abbasi N, et al. Global, regional, and national incidence, prevalence, and years lived with disability for 354 diseases and injuries for 195 countries and territories, 1990-2017: a systematic analysis for the Global Burden of Disease Study 2017. The Lancet. 2018;392(10159):1789-858.

2. Levey AS, Levin A, Kellum JA. Definition and classification of kidney diseases. American Journal of Kidney Diseases. 2013;61(5):686-8.

3. Kellum JA, Lameire N, Aspelin P, Barsoum RS, Burdmann EA, Goldstein SL, et al. Kidney disease: improving global outcomes (KDIGO) acute kidney injury work group. KDIGO clinical practice guideline for acute kidney injury. Kidney international supplements. 2012;2(1):1-138.

4. Levey AS, Becker C, Inker LA. Glomerular filtration rate and albuminuria for detection and staging of acute and chronic kidney disease in adults: a systematic review. Jama. 2015;313(8):837-46.

5. Kiggundu B, Kalyesubula R, Ssinabulya i, Achilles K, Emmanuel S. Chronic Kidney Disease among Hypertensive Patients Attending a National Referral Hospital in Uganda Journal of Urology and Renal Diseases. 2017;144.

6. Kalyesubula R, Nankabirwa JI, Ssinabulya I, Siddharthan T, Kayima J, Nakibuuka J, et al. Kidney disease in Uganda: a community based study. BMC nephrology. 2017;18(1):1-9.

7. Bagasha P, Nakwagala F, Kwizera A, Ssekasanvu E, Kalyesubula R. Acute kidney injury among adult patients with sepsis in a low-income country: clinical patterns and short-term outcomes. BMC nephrology. 2015;16(1):1-7.

8. Odongo P, Wanyama R, Obol JH, Apiyo P, Byakika-Kibwika P. Impaired renal function and associated risk factors in newly diagnosed HIV-infected adults in Gulu Hospital, Northern Uganda. BMC nephrology. 2015;16(1):1-7.

9. Myers GL, Miller WG, Coresh J, Fleming J, Greenberg N, Greene T, et al. Recommendations for improving serum creatinine measurement: a report from the Laboratory Working Group of the National Kidney Disease Education Program. Clinical chemistry. 2006;52(1):5-18.

10. Matsushita K, Mahmoodi BK, Woodward M, Emberson JR, Jafar TH, Jee SH, et al. Comparison of risk prediction using the CKD-EPI equation and the MDRD study equation for estimated glomerular filtration rate. Jama. 2012;307(18):1941-51.

11. Levey AS, Stevens LA, Schmid CH, Zhang Y, Castro III AF, Feldman HI, et al. A new equation to estimate glomerular filtration rate. Annals of internal medicine. 2009;150(9):604-12.

12. Retnakaran R, Cull CA, Thorne KI, Adler Al, Holman RR. Risk factors for renal dysfunction in type 2 diabetes: UK Prospective Diabetes Study 74. diabetes. 2006;55(6):1832-9. 
13. Yokoyama H, Sone $H$, Oishi M, Kawai K, Fukumoto $Y$, Kobayashi $M$, et al. Prevalence of albuminuria and renal insufficiency and associated clinical factors in type 2 diabetes: the Japan Diabetes Clinical Data Management study (JDDM15). Nephrology Dialysis Transplantation. 2009;24(4):1212-9.

14. Noubiap JJN, Naidoo J, Kengne AP. Diabetic nephropathy in Africa: A systematic review. World Journal of Diabetes. 2015;6(5):759.

15. Nyende L, Kalyesubula R, Sekasanvu E, Byakika-Kibwika P. Prevalence of renal dysfunction among HIV infected patients receiving Tenofovir at Mulago: a cross-sectional study. BMC nephrology. 2020;21(1):1-6.

16. Kalyesubula R, Hau JP, Asiki G, Ssebunya B, Kusemererwa S, Seeley J, et al. Impaired renal function in a rural Ugandan population cohort. Wellcome open research. 2018;3.

17. Astor BC, Matsushita K, Gansevoort RT, Van Der Velde M, Woodward M, Levey AS, et al. Lower estimated glomerular filtration rate and higher albuminuria are associated with mortality and endstage renal disease. A collaborative meta-analysis of kidney disease population cohorts. Kidney international. 2011;79(12):1331-40.

18. Weiner DE, Tighiouart H, Amin MG, Stark PC, MacLeod B, Griffith JL, et al. Chronic kidney disease as a risk factor for cardiovascular disease and all-cause mortality: a pooled analysis of communitybased studies. Journal of the American Society of Nephrology. 2004;15(5):1307-15.

19. Kilonzo SB, Seiffudin AT, Bakshi FA, Gunda DW. Renal dysfunction among adult patients in Mwanza, Tanzania: prevalence, outcomes and associated factors. Tanzania Journal of Health Research. 2016;18(3).

\section{Figures}




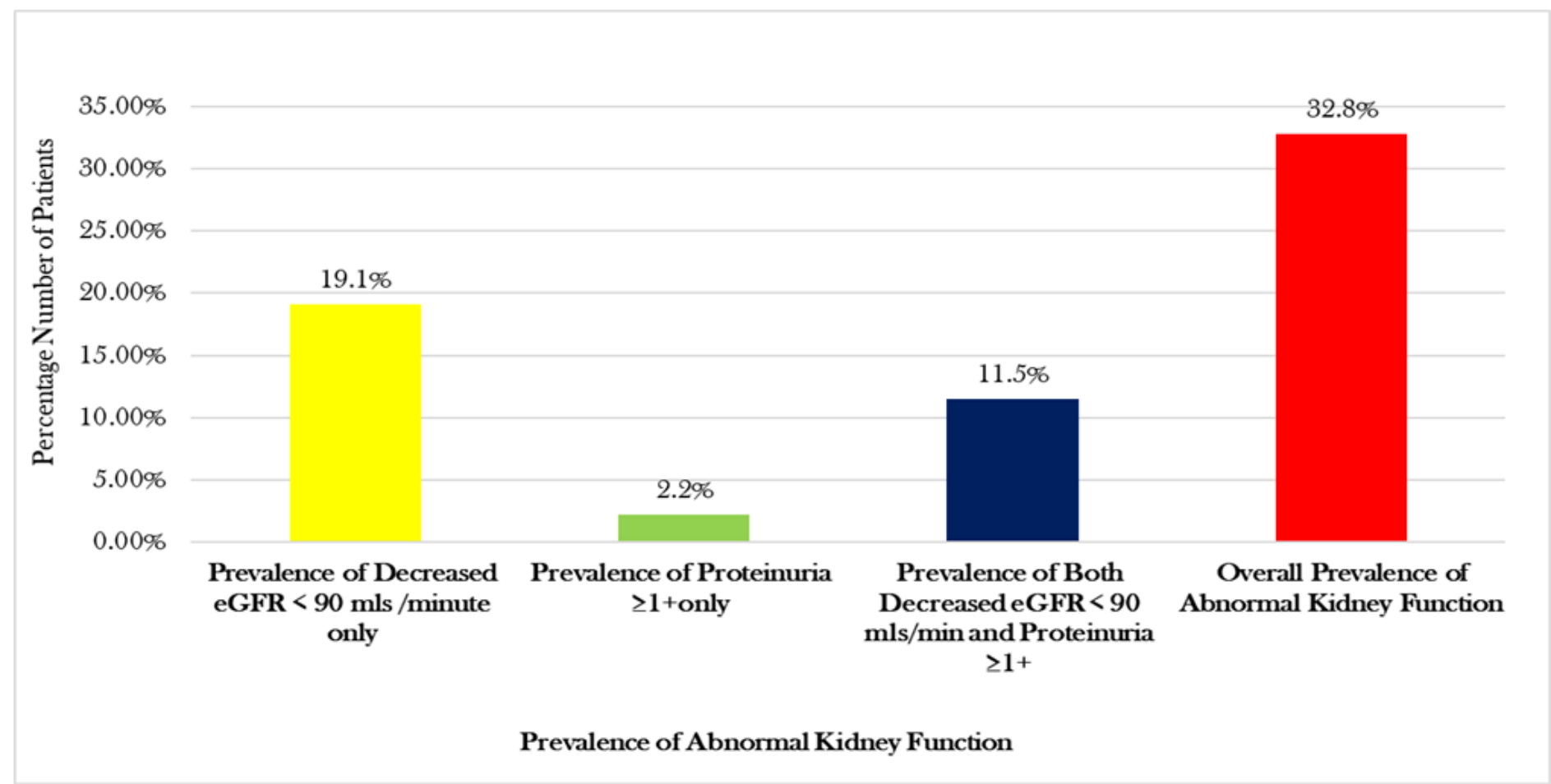

\section{Figure 1}

Prevalence of Abnormal Kidney function. Bar chart shows prevalence of abnormal kidney function among the 357 patients enrolled in the study. The overall prevalence of abnormal kidney function was high at $32.8 \%$ while prevalence of proteinuria of $\geq+1$ only was low at $2.2 \%$. N=Number of patients enrolled in the study.

\section{Supplementary Files}

This is a list of supplementary files associated with this preprint. Click to download.

- Additionalfile1StudyDataSet.xlsx 